\title{
Analysis of demand response for datacenter energy management using GA and time-of-use prices
}

\author{
Berk Celik*, Gustavo Rostirolla* ${ }^{* \dagger}$, Stephane Caux*, Paul Renaud-Goud ${ }^{\dagger}$, Patricia Stolf ${ }^{\dagger}$ \\ *LAPLACE, Université de Toulouse, France \\ \{berk.celik, gustavo.rostirolla, caux\}@laplace.univ-tlse.fr \\ ${ }^{\dagger}$ IRIT, Université de Toulouse, France \\ \{paul.renaud.goud, stolf\}@irit.fr
}

\begin{abstract}
This paper presents an energy management algorithm for a grid-connected datacenter to provide cost-efficient consumption opportunity in a Demand-Response (DR) program. The aim of this study is to schedule controllable loads of the datacenter based on on-peak and off-peak periods of different time-varying electricity prices. The electricity consumption of the datacenter is modeled with two types of workload: batch and service jobs. Service jobs are executed as soon as submitted (as non-controllable) while batch jobs can be executed over the time horizon (as controllable). Moreover, the power demand of the batch jobs can be modified by providing less/more processing resources during their execution (degradation mode). Therefore, the batch jobs give flexibility in order to provide participation opportunity to DR program for the datacenter. In this study, we determine the profitability of different time-of-use electricity prices on datacenter by solving cost-minimization problem using genetic algorithm. Simulation results show that the presented decision algorithm is able to reduce the electricity cost by participating in DR without decreasing the quality of services.

Index Terms-Datacenters, energy management, demand response, time-of-use price, task scheduling, quality of services
\end{abstract}

\section{INTRODUCTION}

The rapid growth of the electricity demand due to high population and new consumption devices (personal electronics, electric vehicles, etc.) starts to threat the reliability of electricity grid. The increased power demand raises the stress over the grid and can create critical conditions on the power system such as contingencies and blackouts. Therefore, Independent System Operator (ISO) should respond to the changes either reinforcing the electricity grid (which requires high investment) or deploying Demand Response (DR) programs [1].

DR becomes essential approach for load planning (i.e., load shifting, shedding) and a promising tool that enables altering the energy consumption of the entities in all electricity sectors: residential, commercial, industrial and transportation. According to [2], DR objective is: changes in electricity usage by end-use customers from their normal consumption patterns in response to changes in the price of electricity, or incentive payments designed to induce lower electricity use at time of high wholesale market prices or when system reliability is jeopardized. On other words, DR encourages end-users to change their consumption habits by offering financial benefits via incentives and/or price signals, thus customers gain active role by modifying their own consumption to increase their welfare (reducing electricity bill) with DR, while ISO maintains supply-demand balance efficiently.

DR is to be implemented on various grid-interactive buildings including datacenters. The escalating demand in Internet access boosts the energy usage on Internet Technology (IT); especially, the proportion of internet users has steadily increased to more than $90 \%$ in many developed countries [3]. This increase translates into a significant increase in the usage of datacenters, while in 2011 they were already consuming more energy than the entire United Kingdom [4]. These facilities are now called "the factories of the digital age". Some authors also predict a continuous growth of $7 \%$ annually through 2030 in the electrical energy usage for manufacture and powering of IT and communication devices [5]. Therefore, datacenters become major consumers in electricity grid and DR is expected to play an important role to operate IT sources efficiently with demand dispatching.

In this paper, we present an energy management algorithm that reduces the cost of electricity consumption in the datacenter using different time-of-use pricing. The datacenter earns financial profit by executing jobs over time without jeopardizing Quality of Services (QoS). In this work, the consumption of electric power associated to workload of datacenter is modeled by classifying workloads in two groups: non-controllable (service jobs) and controllable (batch jobs). Service can be seen as a program that is always executing in the cloud environment receiving a variable amount of requests over time (which results in a proportional power consumption). On the other hand, batch tasks can be represented as an amount of work (load) that needs to be executed between a time period (defined by release and due date).

The rest of the paper is organized as follows: Section II introduces DR; Section III describes the workload and electricity price; Section IV introduces the base control; Section V formulates the optimization problem; Section VI presents the simulation results; and Section VII concludes the paper.

\section{RELATED WORK}

DR programs are classified in two groups: price-based and incentive-based programs [1]. In the first group, customers respond to time-varying pricing signals such as Time-Of-Use (TOU), real-time price, etc., and schedule their loads to benefit from low prices. In the second group, energy providers propose 
incentives to customers for influencing their consumption pattern when the grid reliability is compromised. According to deployed DR programs, customers participate and provide DR services in return of gaining economical profits.

DR is widely studied in residential areas in order to reduce the electricity bill of smart homes [6] using various optimization methods: linear programming [7], mixed integer nonlinear program [8], dynamic programming [9] and heuristic methods such as particle swarm optimization [10]. For commercial buildings, different price values are determined by providers. The reason is that customers of two sectors have different consumption pattern, hence on-peak and off-peak hours/rates are located/determined differently. In literature, DR is studied on various commercial grid-interactive buildings such as hotels [11], universities [12], and typical office buildings [13]. In this area, the studies are mostly based on controlling the indoor temperature according to inhabitant comfort.

On the other hand, datacenters are able to provide additional flexibility by allocating their workloads over the time horizon. Therefore, they can provide valuable DR services for the electricity grid while reducing the cost of energy consumption. Accordingly, in the literature, various methods using DR are proposed as a way to increase datacenter revenues. In [14], the dynamic pricing which is determined based on the aggregated consumption of datacenter, is used with robust optimization for the workload control of the datacenter. In [15], the datacenter participates into emergency DR using "locational" marginal pricing (in form of real-time price). In [16], the energy management reduces the cost of using generation resources with mixed integer linear programming.

In previous work [17], the scheduling of workloads (considering only batch jobs) under a power envelope is studied without connection to the power grid. The power envelope refers to renewable energy production but the power delivery limitation may originate from various reasons (i.e., transformer rating, line capacity limit). The aim of the optimization problem is to allocate IT resources so that QoS degradation is minimized.

However, this paper does not allow degradation on workloads of grid-connected datacenter and aims to minimize the electricity cost using a Genetic Algorithm (GA). The computational complexity of the optimization problem (coming from high-time resolution, IT constraints and high number of decision parameters) is solved with time resolution shifting method. The time-shifting method enables optimizing with low-time resolution (which reduces the computation time) and uses the actual resolution while determining the electricity profiles. Lastly, the different time-varying electricity prices from different providers are used to evaluate and compare the performance of DR control without degrading QoS.

\section{System Model}

\section{A. Time-of-use electricity prices}

In this study, the price-based DR model is deployed using Time-Of-Use (TOU) prices from two different utility companies: ConEdison [18] and Salt River Project [19]. The utilities propose different TOU pricing for summer and winter seasons. In the literature, TOU prices are partitioned into periods: traditionally off-peak, shoulder and on-peak. These periods are rated differently: the highest for on-peak, the average for shoulder and the lowest for off-peak. Accordingly, two blocks TOU for ConEdison and three blocks TOU for SRP are given in Table I. It should be noted that TOU prices are used for commercial buildings which consumes more than $5 \mathrm{~kW}$.

TABLE I

CONEDISON AND SRP ELECTRICITY PRICE

\begin{tabular}{|c|c|c|c|c|}
\hline Source & Tariff & Period & Time & $\mathrm{c} \$ \backslash \mathrm{kWh}$ \\
\hline \multirow{4}{*}{ ConEdison } & \multirow{2}{*}{ Winter } & off-peak & $(00: 00-08: 00)$ & 1.54 \\
\hline & & on-peak & $(08: 00-24: 00)$ & 8.07 \\
\hline & \multirow{2}{*}{ Summer } & off-peak & $(00: 00-08: 00)$ & 1.54 \\
\hline & & on-peak & $(08: 00-24: 00)$ & 21.80 \\
\hline \multirow{7}{*}{ SRP } & \multirow{4}{*}{ Winter } & & $(00: 00-05: 00)$ & \\
\hline & & off-peak & $(09: 00-17: 00)$ & 5.02 \\
\hline & & b l & (21:00-24:00) & 1026 \\
\hline & & on-peak & $(05: 00-09: 00)$ & 11.41 \\
\hline & \multirow{3}{*}{ Summer } & off-peak & $\begin{array}{l}(00: 00-11: 00) \\
(23: 00-24: 00)\end{array}$ & 5.48 \\
\hline & & shoulder & $\begin{array}{l}(11: 00-14: 00) \\
(19: 00-23: 00)\end{array}$ & 10.50 \\
\hline & & on-peak & $(14: 00-19: 00)$ & 15.41 \\
\hline
\end{tabular}

\section{B. Electricity Consumption}

The power consumption of the datacenter is determined similarly compared to the model in [20] using two parameters: active node number (often called static power) and the usage of the cores on each computing node (often called dynamic power). A node refers to a server composed by several cores which are individual processing units in the same processor. Accordingly, the total dynamic power consumption of workloads in the datacenter is calculated with:

$$
P_{w}^{c}(t)=\left(\sum_{s=1}^{S}\left(\sum_{i_{s}=1}^{I_{S}} P_{i_{s}}^{s}(t)\right)\right)+\left(\sum_{b=1}^{B}\left(\sum_{i_{b}=1}^{I_{B}} P_{i_{b}}^{b}(t)\right)\right)
$$

where, $s$ and $b$ are indexes of service and batch jobs; $i_{s}$ and $i_{b}$ are phase index of service and batch phases; $S$ and $B$ total number of services and batches; $I_{S}$ and $I_{B}$ are the total phase number of service and batch jobs; $P_{i_{s}}^{s}(t)$ and $P_{i_{b}}^{b}(t)$ are the power consumption of service and batch jobs; and $P_{w}^{c}(t)$ is the total power consumption of service and batch jobs. Using (1), the total power consumption of the datacenter is determined including active node consumption by:

$$
P_{c}(t)=\left(\sum_{n=1}^{N} P_{\text {node }} \cdot \omega_{n}(t)\right)+P_{w}^{c}(t), \quad \omega_{n} \in\{0,1\}, t \in T
$$

where, $P_{c}(t)$ is total power consumption of datacenter; $n$ is the index of node and $N$ is the total node number; $P_{\text {node }}$ is the constant active consumption; and $\omega_{n}$ is the operation binary variable of the node $(0$ : the node is OFF, 1 : the node is 
ON). It should be noted that, the energy consumption of other equipments (i.e. cooling devices) are not considered, and the network with RAM usages are neglected in this study.

\section{Workload Modeling}

The service jobs are considered as the critical workloads. They are executed as soon as arrived, hence the electric power should be provided for service load execution directly. On the other hand, the batch jobs are flexible and can be shifted to later hours or receive less/more resources than requested.

The existing scheduling approaches for workloads are considering fixed constant resource consumption (i.e., percent CPU usage) as single-phase. However, a workload can perform varying resource consumption over several time as multiphase [21]. In this work, the power consumption of batch and service jobs are formulated using multi-phase model as:

$$
\begin{gathered}
P_{i_{s}}^{s}(t)=n_{s} \cdot P_{c p u} \cdot \psi_{i_{s}}^{s} \cdot \omega_{i_{s}}^{s}(t) \\
\psi_{s_{i}}^{s} \subseteq[0,1], \omega_{s_{i}}^{s} \in\{0,1\}, t \in T \\
P_{i_{b}}^{s}(t)=n_{b} \cdot P_{c p u} \cdot \psi_{i_{b}}^{b} \cdot \omega_{i_{b}}^{b}(t) \\
\psi_{s_{i}}^{s} \subseteq[0,1], \omega_{b_{i}}^{b} \in\{0,1\}, t \in T
\end{gathered}
$$

where, $n_{s}$ and $n_{b}$ are the required core number by service and batch jobs; $P_{c p u}$ is the power consumption of full CPU usage; $\psi_{i_{s}}^{s}$ and $\psi_{i_{b}}^{b}$ are the percentage of CPU usage of service and batch jobs; and $\omega_{i_{s}}^{s}$ and $\omega_{b_{i}}^{b}$ are the operation binary variable of the service and batch jobs (0: task is OFF, 1: task is ON).

\section{BASE CONTROL}

The base control is considered as a reference case for the performance evaluation of the DR control. In this case, the batch jobs are executed as soon as they are submitted. In this study, service and batch jobs are placed to nodes based on the available CPU gap in cores. All jobs are placed firstly to most occupied cores until there is no enough CPU gap left for the next arriving job. Therefore, the active cores are efficiently utilized (close to $100 \%$ CPU), and the unused ones are powered off. In order to have a fair comparison between base and DR control algorithm, jobs are placed using same principle. Accordingly, the cost of electricity consumption of the datacenter is determined in base control with:

$$
C_{b}^{o}=\sum_{t=1}^{T} P_{c}(t) \cdot \lambda(t) \cdot \triangle t
$$

where, $C_{b}^{o}$ is the original cost of electricity consumption, $\lambda(t)$ is TOU price and $\triangle t$ is the simulation time interval.

\section{Problem Formulation}

In this section, the optimization problem that is solved by the controller is formulated. Based on the electricity price $\lambda(t)$, the controller determines the best start time for execution and CPU usage rating of cores for the batch jobs over the time horizon $[0, T]$. Therefore, in order to determine the optimal execution time, the user preferences (referred as due date) are taken into account. Due date is the last acceptable time for finishing the execution of the batch job for clients. Hence, the controller should not violate the due date of batch jobs and complete all before their deadline. Therefore, the due date constraint is formulated as follows:

$$
\left[t_{b, i_{b}}^{r s}, t_{b, i_{b}}^{r e}\right] \subseteq\left[t_{b, i_{b}}^{s}, t_{b, i_{b}}^{e}\right]
$$

where, $t_{b, i_{b}}^{r s}$ and $t_{b, i_{b}}^{r e}$ are the rescheduled start and end times of batch; and $t_{b, i_{b}}^{s}$ and $t_{b, i_{b}}^{e}$ are the release time and due date of the batch. Additionally, due to multi-phase modeling (as in Section III-C), the batch job is subdivided into small phases (or considered as small jobs). Each phase can be executed with different CPU rating $(0-100 \%)$ with delays after execution of the previous phase. However, phases are not completely independent from each other since each phase should wait until previous phase finishes its operations. Accordingly, phases of the batch job should be executed synchronously using given constraint as below:

$$
t_{b, i_{b}-1}^{r e}<t_{b, i_{b}}^{r s}
$$

Additionally, the controller redetermines CPU rating for each batch phases. Each phase can operate at different CPU rate, hence it is not mandatory to follow previous phase operating characteristic during the execution. Therefore, phases of each job can operate according to:

$$
\psi_{b, i_{b}}^{r b} \subseteq[0,1], \quad i_{b} \in I_{B}, b \in B
$$

where, $\psi_{b, i_{b}}^{r b}$ is the redetermined CPU rate of the batch phase. Finally, the rescheduled consumption profile of the datacenter is determined in order to minimize the electricity cost by calculating the input variables $t_{i_{b}}^{r e}$ and $\psi_{i b}^{r b}$ using (1) - (4) according to:

$$
\begin{aligned}
& \min \left\{C_{b}^{r}=\sum_{t=1}^{T} P_{c}(t) \cdot \lambda(t) \cdot \Delta t\right\} \\
& \text { subject to }(6),(7),(8)
\end{aligned}
$$

where, $C_{b}^{r}$ is the rescheduled cost of the datacenter (recall from (5); $\lambda(t)$ is TOU price; and $\triangle t$ is the time interval.)

In this work, GA is used for solving the formulated optimization problem. We preferred to use GA which is used to solve similar types of optimization problems, due to ability to find near optimal solution in acceptable time duration in a large problem. In deployed GA algorithm, each chromosome represents CPU ratings and rescheduling start-time of the batch jobs. By two points crossing-over (with linear-bias) and mutation , GA solves the (9). The parameters of the GA are chosen arbitrary: population size $=100$, bias rate $=1.4$, mutation rate $=0.01$, $\max$ generation $=500,000$. The first population of the GA is generated randomly. It should be noted that any type of optimization method can be used for solving the given optimization problem in (9).

\section{Simulation Results}

\section{A. Simulation Platform}

The simulations are performed for four cases using different TOU pricing given in Section III-A and compared with the base control given in Section IV. We assume that the datacenter is formed by $N=200$ nodes, and each node has eight 

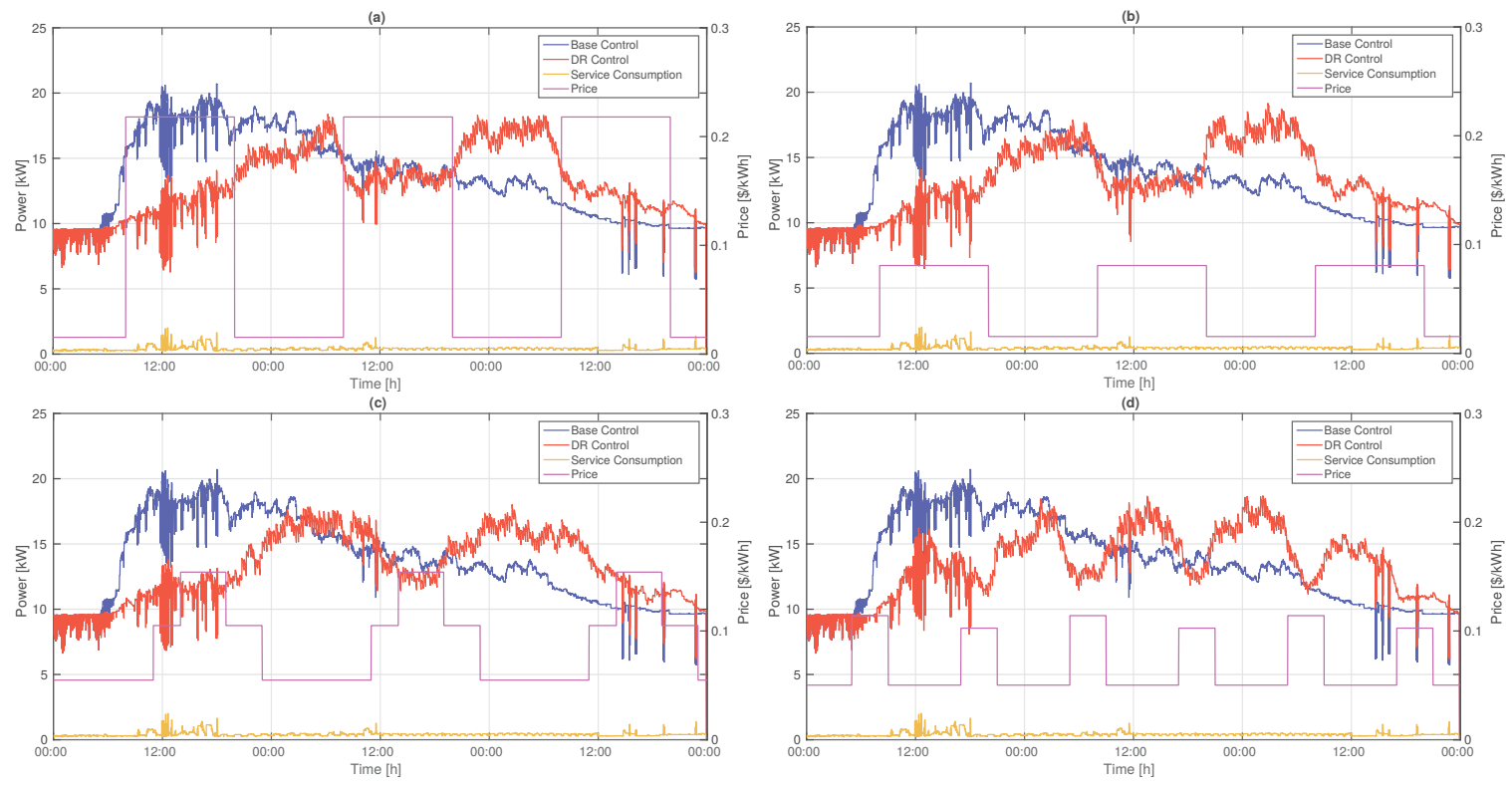

Fig. 1. Electricity consumption profiles of datacenter. (a) ConEdison results for summer season, (b) ConEdison results for winter season, (c) SRP results for summer season, (d) SRP results for winter season.

CPU cores that executes $S=8,058$ service and $B=2,506$ batch jobs. The simulated day is divided into $T=86,400$ time intervals with $1 \mathrm{~s}$ resolution, and simulations are performed for consecutive three days. The power consumption of $100 \% \mathrm{CPU}$ usage of a single core is taken $P_{c p u}=16.25 \mathrm{~W}$ and the active node power consumption is assumed to be $P_{\text {node }}=70 \mathrm{~W}$.

However, it should be noted that one-second time resolution creates high computation burden, hence the time-resolution is reduced to 15-minutes while solving the optimization problem. To change the time resolution, first the aggregated consumption profile is created using service jobs, and the maximum value is considered as the consumption of the datacenter at each 15 minutes interval. After that, the start times and due dates of batch jobs are converted to 15-minutes resolution. For instance, if a batch is released at $t=74,573$ seconds, it is released at $t=83$ on new time resolution according to $\left(\frac{74573 s}{15 m \times 60 s}\right)$. In the end of the optimization, all determined results are converted back with same principle, and the electricity profiles are determined with one-second resolution.

For a more accurate representation of a datacenter, we generated jobs called phase-based [21] as described in Section III-C. For the batch workload, the concept of phases is included using a Google based workload generator [22]. It consists in a normal distribution for the consumed CPU rating. The service jobs are based on traces from [23], where the business-critical workloads are studied.

The optimization problem is solved using GA with given setup parameters in Section V. The modeling of service and batch jobs are performed in JAVA, and then the optimization problem is programmed in c++ (objective functions, constraints and GA) compiling with gnu-c++-compiler. Lastly, the optimization problem is solved on a Linux-server with an Intel Xeon Gold@2.10 GHz processor and 16 GB RAM.

\section{B. Performance Evaluation}

The power consumption profiles are given in Fig. 1. As seen, the consumption profiles are impacted by TOU prices differently. ConEdison winter and summer pricing have similar effect on the consumption profile due to same off-peak and on-peak periods. The electrical consumption of the datacenter is shifted to late night and/or early morning hours using ConEdison prices. The DR control creates another high peak consumption at off-peak period. On the other hand, SRP winter effects significantly compared to ConEdison prices while SRP summer has similar effect over the optimization algorithm. The reason is that SRP summer has almost similar off-peak and on-peak periods compared to ConEdison prices.

In Fig. 2, the cost of electricity consumption and the financial efficiency results are given. The efficiency parameter " $\eta$ " is simply calculated using (10). The highest costs are $119.92 \$$ on base control and $106.03 \$$ on DR control for ConEdison summer which leads to a highest efficiency of $11.58 \%$. The minimum costs are gathered $49.06 \$$ and $43.93 \$$ for ConEdison winter on base and DR controls, respectively. However, the lowest efficiency is recorded with SRP winter with $4.53 \%$ reduction. The reason, why the lowest efficiency and the lowest cost are not the same, is the difference between on-peak and off-peak prices. This difference is higher on ConEdison summer than SRP winter. On the other hand, we can expect that SRP summer should provide better efficiency than ConEdison winter based on difference between on-peak and off-peak prices. However, it should be noted that the workload scheduling does not only depend on the pricing tariffs, it is also related to releasing time and due dates of batch jobs and activated node numbers.

$$
\eta=\left(\frac{C_{b}^{o}-C_{b}^{r}}{C_{b}^{o}}\right) \times 100
$$



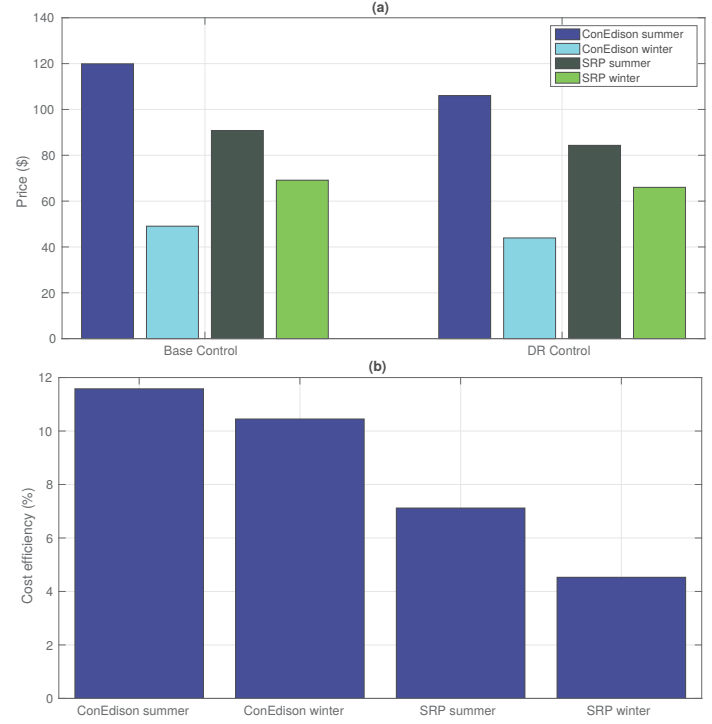

Fig. 2. Cost analysis of the TOU prices. (a) Costs of electricity consumption, (b) Cost reduction efficiencies of DR control.

Lastly, the computation time for solving optimization with GA is recorded between $2-5$ hours for 3 days of workload. The performance of the GA depends on various parameters: setup parameters of GA (i.e., population size), price structure, workload parameters (i.e., release time), workload number and datacenter structure (nodes, cores, etc.).

\section{CONCLUSION}

This paper presented an energy management algorithm for electricity consumption of the datacenter by analyzing the effect of DR using time-varying electricity prices and a mixed of controllable and non controllable load (batches and services). The algorithm aims at reducing the cost of electricity by scheduling load to the off-peak periods and reducing the electricity consumption of datacenter without jeopardizing QoS. Results showed that the proposed DR control is able to decrease the electricity bill of the datacenter using different TOU prices without impacting the QoS. However, it should be noted that the level of cost reduction and efficiency of the algorithm are associated to various factors such as on-peak and off-peak time periods, release times, due dates, level of flexibility in both side (larger effort relaxing provider price and IT user demand).

\section{ACKNOWLEDGMENT}

This work was supported by the French ANR project DATAZERO (ANR-15-CE25-0012).

\section{REFERENCES}

[1] B. Celik, R. Roche, S. Suryanarayanan, D. Bouquain, and A. Miraoui, "Electric energy management in residential areas through coordination of multiple smart homes," Renewable and Sustainable Energy Reviews, vol. 80 , pp. $260-275,2017$.

[2] U.S. Department of Energy. Benefits of demand response in electricity markets and recommendations for achieving them. Available: https://goo.gl/26Mqbt (Accessed Mar. 2019).
[3] M. Salahuddin and K. Alam, "Information and communication technology, electricity consumption and economic growth in oecd countries: A panel data analysis," International Journal of Electrical Power and Energy Systems, vol. 76, pp. 185 - 193, 2016.

[4] J. Koomey, "Growth in data center electricity use 2005 to 2010," A report by Analytical Press, completed at the request of The New York Times, p. 9, 2011.

[5] Greenpeace, "Clicking Clean: Who is winning the race to build a green internet?” Greenpeace, Tech. Rep., Jan. 2017.

[6] T. Logenthiran, D. Srinivasan, and T. Z. Shun, "Demand side management in smart grid using heuristic optimization," IEEE Transactions on Smart Grid, vol. 3, no. 3, pp. 1244-1252, Sep. 2012.

[7] A. Agnetis, G. de Pascale, P. Detti, and A. Vicino, "Load scheduling for household energy consumption optimization," IEEE Transactions on Smart Grid, vol. 4, no. 4, pp. 2364-2373, Dec 2013.

[8] D. Setlhaolo, X. Xia, and J. Zhang, "Optimal scheduling of household appliances for demand response," Electric Power Systems Research, vol. 116 , pp. $24-28,2014$.

[9] C. Keerthisinghe, G. Verbi, and A. C. Chapman, "A fast technique for smart home management: Adp with temporal difference learning," IEEE Transactions on Smart Grid, vol. 9, no. 4, pp. 3291-3303, July 2018.

[10] D. Fuselli, F. D. Angelis, M. Boaro, S. Squartini, Q. Wei, D. Liu, and F. Piazza, "Action dependent heuristic dynamic programming for home energy resource scheduling," International Journal of Electrical Power \& Energy Systems, vol. 48, pp. 148 - 160, 2013.

[11] A. P. Rogers and B. P. Rasmussen, "Opportunities for consumerdriven load shifting in commercial and industrial buildings," Sustainable Energy, Grids and Networks, vol. 16, pp. 243 - 258, 2018.

[12] S. Bracco, M. Brignone, F. Delfino, F. Pampararo, M. Rossi, G. Ferro, and M. Robba, "An optimization model for polygeneration microgrids with renewables, electrical and thermal storage: Application to the savona campus," in 2018 IEEE International Conference on Environment and Electrical Engineering and 2018 IEEE Industrial and Commercial Power Systems Europe (EEEIC / I CPS Europe), June 2018, pp. 1-6.

[13] S. Aghniaey and T. M. Lawrence, "The impact of increased cooling setpoint temperature during demand response events on occupant thermal comfort in commercial buildings: A review," Energy and Buildings, vol. 173, pp. $19-27,2018$.

[14] T. Chen, Y. Zhang, X. Wang, and G. B. Giannakis, "Robust geographical load balancing for sustainable data centers," in 2016 IEEE International Conference on Acoustics, Speech and Signal Processing (ICASSP), March 2016, pp. 3526-3530.

[15] R. Wang, N. Kandasamy, C. Nwankpa, and D. R. Kaeli, "Datacenters as controllable load resources in the electricity market," in 2013 IEEE 33rd International Conference on Distributed Computing Systems, July 2013, pp. 176-185.

[16] C. Gu, H. Huang, and X. Jia, "Green scheduling for cloud data centers using ESDs to store renewable energy," in 2016 IEEE International Conference on Communications (ICC), May 2016, pp. 1-7.

[17] S. Caux, P. Renaud-Goud, G. Rostirolla, and P. Stolf, "IT optimization for datacenters under renewable power constraint," in Euro-Par 2018: Parallel Processing - 24th Int. Conference on Parallel and Distributed Computing, Turin, Italy, August 27-31, 2018, Proceedings, 2018, pp. 339-351. [Online]. Available: https://doi.org/10.1007/978-3-319-96983$1 \_24$

[18] ConEdison. Time-of-Use Rates. Available: https://goo.gl/BYCqxv (Accessed Nov. 2018).

[19] Salt River Project. Business Time-of-Use Price Plan. Available: https://goo.gl/UgUCmx (Accessed Nov. 2018).

[20] T. Mudge, "Power: A first-class architectural design constraint," Computer, vol. 34, pp. 52-58, 2001.

[21] M. Diouri, G. L. Tsafack Chetsa, O. Gluck, L. Lefèvre, J.-M. Pierson, P. Stolf, and G. Da Costa, "Energy efficiency in HPC: with or without knowledge on applications and services," Int. Journal of High Performance Computing Applications, pp. 232-243, Aug 2013.

[22] G. Da Costa, L. Grange, and I. de Courchelle, "Modeling, classifying and generating large-scale google-like workload," Sustainable Computing: Informatics and Systems, 2018.

[23] S. Shen, V. v. Beek, and A. Iosup, "Statistical characterization of business-critical workloads hosted in cloud datacenters," in 2015 15th IEEE/ACM Int. Symposium on Cluster, Cloud and Grid Computing, May 2015, pp. 465-474. 